\title{
LA IDENTIFICACIÓN DE LAS UNIDADES LÉXICAS INTEGRANTES DE LAS MEDIOESTRUCTURAS SEMÁNTICAS DE LOS VERBOS
}

\begin{abstract}
Mehlberg Martin, La identificación de las unidades léxicas integrantes de las medioestructuras semánticas de los verbos [Identification of lexical units members of the semantic mediostructures of verbs]. Studia Romanica Posnaniensia, Adam Mickiewicz University Press, Poznań, vol. XXXl: 2004, pp. 369-375. ISBN 83-232-1353-4, ISSN 0137-2475.
\end{abstract}

Most verbs exhibit a range of different conventionalized meanings (lexical units), which form the socalled mediostructure of the verb. Based on the model of Lexical Syntax (Subirats, 2001), we will outline a possible identification procedure for these various lexical units. This procedure consists in determining the class of linear projection and of syntactic redundancy to which the lexical unit in question belongs.

\section{INTRODUCCIÓN: EL CONCEPTO DE LA MEDIOESTRUCTURA}

En la presente comunicación vamos a tratar de caracterizar la interrelación entre el significado de los elementos léxicos y su potencial combinatorio sintagmático. Partimos de la hipótesis de que la correlación del significado con el nivel sintagmático nos permite elaborar criterios que posibilitan la descripción de la llamada medioestructura semántica (Wotjak, en prensa). Como medioestructura designamos el conjunto de las microestructuras con la misma forma morfofonológica $\mathrm{o}$, desde un punto de vista más bien lexicográfico, el conjunto de las acepciones que aparece bajo un lema determinado. Las microestructuras constituyen "invariantes semánticas, sememizadas, sistémicas usualizadas [...] y socializadas, es decir, compartidas virtualmente por todos los hablantes de una misma comunidad lingüística y/o comunicativa [...]" (Wotjak, en prensa). Consideramos las microestructuras como unidades básicas de la descripción léxica. Equivalen a las unida- 
des léxicas de Cruse que las define como "union of a lexical form and a single sense" (Cruse, 1986: 77) ${ }^{1}$.

Si partimos aquí de la base de que un verbo puede tener varios significados convencionalizados que establecen una medioestructura semántica, no caracterizamos con esto las relaciones entre las varias unidades léxicas ${ }^{2}$. Para descripciones de índole sincrónico no tiene mucha importancia esta cuestión teórica, ya que es importante que se especifiquen todos los significados que puede adquirir un elemento léxico (cf. Stein, 1999: 114).

En lo que sigue, queremos demostrar que es posible analizar las medioestructuras de verbos en el marco de un modelo diferencial que se basa en las relaciones sintácticas y semánticas entre los elementos del léxico. A partir de tal modelo deberíamos ser capaces de deducir criterios que nos permitan identificar las unidades léxicas (o predicados en el marco del modelo que usamos) que constituyen la medioestructura semántica de un verbo.

\section{EL MODELO DE LA SINTAXIS LÉXICA}

Como base teórica y metodológica para conseguir este propósito, se ofrece el modelo de la Sintaxis Léxica (Subirats 2001) que podemos caracterizar como lexicalista, es decir, que explica el funcionamiento de las lenguas naturales desde una perspectiva léxica ${ }^{3}$. La hipótesis central se refiere a la transmisión de la información en las lenguas naturales: esta transmisión se basa en la redundancia sintáctica. La redundancia se manifiesta en las restricciones que les imponen los predicados $^{4}$ a las construcciones sintácticas en las que pueden aparecer. En la medida en que estas restricciones son únicas para cada predicado, se establecen clases de redundancia sintáctica integradas por un sólo elemento ${ }^{5}$. Esto significa que, al enumerar el conjunto único de estas restricciones léxicamente condicionadas, podemos delimitar los predicados sintácticamente. A continuación vamos a

'También Apresjan (2000) y Johnson et al. (2002), entre otros, ven la unidad léxica como unidad básica de la descripción léxica.

${ }^{2}$ Esa caracterización explicaría cómo ha surgido la medioestructura en cuestión: en el caso de la homonimia a causa de mutaciones fonéticas en las formas de dos unidades léxicas distintas, en el caso de la polisemia, a causa de la convencionalización de transposiciones y extensiones conceptuales. Sin embargo, hay otra causa, hasta ahora poco considerada, para la formación de medioestructuras, a saber, los préstamos. Por ejemplo, en el alemán ha habido una ampliación en la medioestructura del verbo realisieren porque ha entrado en la lengua un préstamo del inglés, es decir, se formó una unidad léxica nueva (realisieren 'darse cuenta') dentro de la medioestructura ya existente.

${ }^{3}$ El modelo se basa en la Teoría de Predicados de Harris (1991).

${ }^{4}$ Se trata de predicados verbales, nominales, adjetivales, preposicionales y adverbiales.

${ }^{5}$ Esto implica que no se puede desvincular la sintaxis del léxico y que, en este sentido, no existen reglas gramaticales independientes del léxico. 
esbozar los componentes básicos del modelo en tanto que nos sirven para extraer criterios para la identificación de predicados o unidades léxicas.

La capacidad de las lenguas naturales de transmitir información es resultado de la capacidad de poder establecer jerarquías de dependencia. Estas jerarquías se forman a través de predicados que operan sobre sus argumentos. La (im-) posibilidad de establecer una jerarquía de predicación a partir de los elementos léxicos ya induce una primera partición en el léxico, a saber, en los elementos léxicos que no poseen un requerimiento argumental, es decir, que no son capaces de establecer una jerarquía de dependencia (argumentos de nivel cero $N$ ) y en los elementos léxicos que poseen un requerimiento argumental (predicados $P$ ). La definición de las propiedades de dependencia de un predicado se fundamenta en las propiedades de dependencia de los elementos léxicos con los que puede aparecer para formar una oración ${ }^{6}$. Podemos distinguir los predicados de primer nivel $P_{\text {...n... }}$ (que requieren exclusivamente $N$ para formar una oración) y los de segundo nivel $P_{\ldots \ldots} \ldots$ (que requieren por lo menos a un $P$ para formar una oración). A partir de relaciones de equivalencia se induce una partición en tres clases y sucesivamente en subclases dentro de estas clases. Obtenemos las siguientes clases de dependencia:

\begin{tabular}{|l|}
\hline Elementos léxicos de nivel cero $(\operatorname{argumentos} N)$ \\
\hline Elementos léxicos de primer nivel (predicados $P_{\ldots . . .}$ con subclases $\left.P_{n,}, P_{n n}, P_{n n n}\right)^{7}$ \\
\hline Elementos léxicos de segundo nivel (predicados $P_{\ldots, \ldots \ldots}$ con subclases $P_{p}, P_{n p}, P_{p n}$ etc.) \\
\hline
\end{tabular}

La relación de dependencia entre un predicado y una clase de argumentos todavía no especifica los elementos que aparecen realmente en las oraciones. Sólo podemos constatar que para todos los elementos de la clase existe una probabilidad positiva de aparición como argumento. Es obvio que la probabilidad de aparición como argumento de un determinado predicado no puede ser la misma para todos los elementos de la clase, por lo tanto, decimos que los elementos que tienen comparativamente más posibilidades de aparecer como argumentos, constituyen la selección del predicado. Existe una relación de complementariedad entre un predicado y su selección: por un lado, el significado de un predicado determina su selección, por otro lado, la selección ayuda a delimitar el significado de un predicado ${ }^{8}$.

\footnotetext{
${ }^{6}$ Nótese que el requerimiento argumental de un predicado se define en función de las propiedades de dependencia de los argumentos. No se recurre a clases de argumentos que se basan en criterios morfológicos o distribucionales. Por lo tanto sería mejor designar csta clase de dependencia como $A$ (y no como $N$ que es el símbolo que usa Subirats y que hemos adoptado aquí), ya que no se presta tanto a confundir la clase de dependencia con la clase distribucional de los nombres. Por cierto, todos los $\operatorname{argumentos} N$ son nombres (por ejemplo, luna, Juan, manzana), pero no todos los nombres pertenecen a la clase $N$. Sobre todo nombres abstractos, por ejemplo guerra, aversión, pertenecen a la clase $P$.

${ }^{7}$ La representación del requerimento argumental de un predicado refleja el número de argumentos que admite, el tipo de argumentos $(N \circ P)$, y el orden en que aparecerían de acuerdo con su posición canónica en las oraciones de la base (Bobes, 2002: 13).

${ }^{8}$ También podríamos decir que posibilita la disambiguación.
} 
Ahora podemos caracterizar mejor las oraciones de una lengua: son proyecciones lineales de las relaciones de dependencia que se establecen entre los elementos léxicos. Esa relación de dependencia entre predicados y argumentos está plenamente explícita en las oraciones de la base, que proyectan las jerarquías de predicación siguiendo un orden canónico. A las oraciones de la base no se les ha aplicado ninguna transformación que pueda ocultar las relaciones de predicación o crear ambiguiedad. Por lo tanto, las oraciones de la base representan proyecciones del léxico y tienen su origen en las propiedades de dependencia y selección de los predicados.

Las proyecciones lineales se usan como representaciones del requerimiento argumental de clases de predicados o predicados concretos y se especifican en ellas los elementos siguientes:

a) tipo del predicado (indicado como clase, por ejemplo, Vpred, Npred, Apred o como la especificación léxica, por ejemplo, visitar),

b) clase de dependencia de los argumentos $(N \circ P)$ marcados con subíndices que indican si son el $1^{\circ}, 2^{\circ}$ o $3^{\circ}$ argumento en la oración de la base,

c) marcadores de argumento (para $N: a$, de, por, con, en etc.; para $P: q u e, s i$ ).

La proyección lineal del requerimiento argumental de los predicados induce otra partición en las clases de dependencia que crea clases de predicados que tienen la misma proyección lineal, por ejemplo, los predicados verbales fracasar y suspirar (Luis fracasó en el examen; Luis suspira por unas vacaciones en las montañas) son ambos $P_{n n}$, sin embargo, proyectan su requerimiento argumental de modo diferente y, por lo tanto, pertenecen a clases distintas $\left(N_{1}\right.$ Vpred en $N_{2} ; N_{1}$ Vpred por $\mathrm{N}_{2}$ ).

Se puede actualizar la información (potencial) de las jerarquías de predicación, que se forman a través del requerimiento argumental y la selección de los predicados, no sólo en las oraciones de la base sino también en otras construcciones sintácticas. Estas realizaciones alternativas de las jerarquías se consideran paráfrasis semánticas porque se mantienen las respectivas jerarquías. Por eso, se derivan de las oraciones de la base mediante transformaciones que consisten en cambios formales de una oración ${ }^{9}$. Las diferencias formales y semánticas en relación a las oraciones de la base son siempre sistemáticas y productivas en el léxico ${ }^{10}$.

Ahora es posible establecer clases de equivalencia de las oraciones con jerarquías de predicación idénticas. Estas clases abarcan las oraciones de la base

${ }^{9}$ Se trata entonces de transformaciones en el sentido harrisiano y no chomskyano: "[...] micntras que, para Chomsky [...] una transformación es una operación abstracta de permutación de un elemento $\alpha$, que se sitúa entre la estructura profunda y la estructura $\mathrm{S}[\ldots]$ aquí las transformaciones corresponden a un dispositivo experimental destinado a poner de manifiesto, a través de frases semántica y morfológicamente análogas, las características sintácticas pertinentes de una estructura detcrminada" (Lamiroy 1991: 15). Otro término que encontramos es alternancias de diátesis.

${ }^{10}$ Pustejovsky se refiere a este cambio semántico en las oraciones derivadas con el término verual polysemy (Pustejovsky, 2002: 567) aunque sería más preciso hablar de grammatical polysemy (Stein, 1999: 115). 
que son proyecciones canónicas del requerimiento argumental de un predicado y las oraciones derivadas mediante transformaciones, que mantienen relaciones formales y semánticas sistemáticas con las oraciones de la base. La posibilidad de aplicar una determinada transformación puede depender de criterios formales de una construcción sintáctica. Así cualquier segundo argumento con las propiedades de pronominalización que caracterizan a los objetos indirectos admite la reduplicación pronominal: Luis presta dinero a Juan. $\rightarrow$ Luis le $e_{i}$ presta dinero a Juan. (Cf. Subirats, 2001: 51). Estas transformaciones no están condicionadas léxicamente. Al contrario, las transformaciones léxicamente condicionadas son aquellas que se pueden aplicar en el caso de que un predicado se encuentre en su dominio de aplicación. Esto constituye una propiedad idiosincrásica del predicado en cuestión. Las transformaciones léxicamente condicionadas ocupan un lugar central dentro del modelo de la Sintaxis Léxica ya que posibilitan la determinación de las clases de redundancia sintáctica que contienen, en general, un sólo elemento del conjunto de todos los predicados de una lengua.

\section{UN PROCEDIMIENTO PARA LA IDENTIFICACIÓN DE UNIDADES LÉXICAS}

Tras la explicación de unos conceptos básicos del modelo, podemos esbozar un procedimiento sistemático que nos permita identificar los predicados con formas idénticas que corresponden a unidades léxicas dentro de la medioestructura semántica de un verbo. Tal procedimiento se basa esencialmente en las clases de predicados que se forman a través de particiones sucesivas en el léxico. Esta manera de proceder está fundamentada en la hipótesis de que existe una correlación sistemática entre la diferenciación formal y semántica de los predicados, es decir, que el significado de un predicado depende tanto de su selección como de sus propiedades formales. Esto implica que no hay dos predicados con las mismas propiedades formales y/o de selección (Subirats, 2001: 22). Podemos interpretar esta característica de las lenguas naturales como un procedimiento para mantener la diferenciación semántica entre todos los predicados. Esto tiene validez tanto para los predicados con formas distintas como para aquellos con formas idénticas. La diferencia consiste en que formas no idénticas facilitan la identificación de los predicados, mientras que los predicados con formas idénticas requieren la identificación por medio de un procedimiento analítico.

El primer paso en el proceso de la identificación debe consistir en determinar las clases de proyección lineal. Las propiedades que usamos para este propósito crean una jerarquía e inducen sucesivamente una partición en el conjunto de los predicados (Cf. Subirats, 2001: 102): 
Partición en el conjunto de todos los predicados en función de diferencias en la forma de recibir las marcas de tiempo (los predicados no verbales reciben las marcas indirectamente en sus verbos de soporte) $\Rightarrow$ Resultado: clases de predicados verbales y no verbales

Partición en las clases que se han formado en la partición anterior en función de las propiedades de dependencia de los argumentos $\Rightarrow$ Resultado: clases de predicados de primer y de segundo nivel $\left(P_{\ldots \ldots \ldots} \ldots P_{\ldots p . . .}\right)$

Partición en las clases que se han formado en la partición anterior en función del requerimiento argumental $\Rightarrow$ Resulatdo: clases de dependencia $\left(P_{n n}, P_{p n}, P_{n n p}\right.$ etc. $)$ $\Downarrow$

Partición en las clases que se han formado en la partición anterior en función de la proyección del requerimiento argumental $\Rightarrow$ Resultado: clases de proyección lineal

La formación de clases de predicados con proyecciones idénticas del requerimiento argumental nos permite determinar las propiedades léxico-sintácticas (o sea, transformacionales y seleccionales) para cada elemento de la clase. Una serie de propiedades va a caracterizar toda la clase, mientras que otras propiedades están condicionadas léxicamente, es decir, son propiedades idiosincrásicas de los predicados de la clase. Por consiguiente, podemos constatar que todos los predicados de la clase se diferencian entre sí. Esas diferencias son de índole semántica (diferentes propiedades de selección, por ejemplo, agentividad o no-agentividad del primer argumento) y sintáctica (diferencias basadas en la posibilidad de la aplicación de una determinada transformación). Las propiedades idiosincrásicas inducen otra partición, esta vez en las clases de proyección lineal. Obtenemos así clases de redundancia sintáctica que posibilitan la diferenciación de un predicado de los otros predicados de la misma clase de proyección lineal. Podemos ilustrar este procedimiento con el siguiente ejemplo: (1) Los críticos celebran mucho a este director; (2) Los padres celebran que Luis haya vuelto a casa; (3) Los padres celebran que Luis ha vuelto a casa.

Para determinar si las diferencias semánticas intuitivas realmente implican la existencia de tres predicados o unidades léxicas diferentes tenemos que analizar primero las propiedades de dependencia: en (1) hay un predicado del tipo $P_{n n}$, en (2) un $P_{n p}$ y en (3) otro $P_{n p}$. Las respectivas proyecciones del requerimiento argumental son: (1) $N_{I}$ celebrar a $N_{2}$, (2) $N_{I}$ celebrar que $P_{2}$ y (3) $N_{1}$ celebrar que $P_{2}$. El hecho de que celebrar pertenezca a dos clases de proyección distintas (Vpred a $N_{2}$ y $N_{1}$ Vpred que $P_{2}$ ) implica que hay por lo menos dos predicados. Como el elemento léxico celebrar en (2) y (3) pertencece a la misma clase de proyección, todavía no podemos aclarar si se trata realmente de dos predicados. Por eso tenemos que 
verificar si pertenecen a diferentes clases de redundancia sintáctica, es decir, tenemos que averiguar las propiededades de selección y transformación que conforman las clases de redundancia (Cf. Subirats, 2001: 115). El hecho de que celebrar seleccione el subjuntivo en (2) y el indicativo en (3) para su argumento oracional, ya demuestra la existencia de dos predicados distintos: Además podemos constatar que celebrar en (2) no admite la pasiva con ser y tampoco una nominalización (Los padres celebran que Luis haya vuelto a casa [pasiva ser] $\rightarrow$ $*$ Este acontecimiento es celebrado por ellos; [Nom deverbal] $\rightarrow$ *la celebración de este acontecimiento), mientras que celebrar en (3) admite estas transformaciones (Los padres celebran que Luis ha vuelto a casa [pasiva ser] $\rightarrow$ Este acontecimiento es celebrado por ellos; [Nom deverbal] $\rightarrow$ la celebración de este acontecimiento). Así hemos comprobado que la medioestructura del verbo celebrar está integrada de tres predicados como mínimo: celebrar 1 'alabar', celebrar 2 'alegrarse' y celebrar ${ }_{3}$ 'festejar'.

\section{REFERENCIAS BIBLIOGRAFICAS}

Apresjan, J. D. (2000), Systematic Lexicography. Oxford: OUP.

Bobes Soler, E. de (2002), Sintaxis léxica de las locuciones verbales predicativas del español. Documento electrónico, LaLI, Universidad Autónoma de Barcelona.

Cruse, A. D. (1986), Lexical Semantics. Cambridge: CUP.

Harris, Z. (1991), A Theory of Language and Information. Oxford: Clarendon.

Johnson, C. R. et al. (2002), FrameNet: Theory and Practice. Documento electrónico, International Computer Science Institute, University of Berkeley.

Lamiroy, B. (1991), Léxico y gramática del español, Barcelona: Anthropos.

Pustejovsky, J. (2002), "Syntagmatic Processes", cn D. A. Cruse et al. (eds.), Lexikologie. BerlinNew York: de Gruyter, 565-570.

Stein, A. (1999), "Describing Verb Semantics in a Type Hierarchy: Disambiguation of Italian Verbs", en P. Saint-Dizier (ed.), Predicative Forms in Natural Language and Lexical Knowledge Bases. Dordrecht: Kluwer, 111-137.

Subirats Rüggeberg, C. (2001), Introducción a la sintaxis léxica del español. Francfort: Vervucrt.

Worjak, G. (en prensa), La medioestructura: acerca de la sememización de 'extensiones conceptuales' (metonimia y metáforas) y su reflejo en el diccionario, en Actas del V Congreso de Lingüística General (León, 5-8 de marzo 2002). 